\title{
POLAR MOTION FROM THE TRACKING OF CLOSE EARTH SATELLITES
}

\author{
KURT LAMBECK \\ Observatoire de Paris, 92-Meudon, France
}

\begin{abstract}
The methods of using close earth satellites for determining the motion of the Earth's axis of rotation are discussed. Particular emphasis is given to the possibility of using laser range observations. Typical error sources in orbit computations are discussed and their influence on the resulting pole positions estimated. Within a few years pole coordinates with an accuracy of about $20 \mathrm{~cm}$ and with a resolution of 1 day or even $12 \mathrm{hr}$ can be routinely expected.
\end{abstract}

The astronomical methods employed for determining the motion of the Earth about its center of mass have some severe limitations when very high accuracies and high resolutions are sought. For example, Guinot (1970) estimates that accuracies in pole positions of 0.01 and in rotational speed of $0.001 \mathrm{~s}$ are possible only for averaging periods of about 5 days. Thus it becomes impossible to observe directly some of the interesting nearly diurnal terms resulting from the free nutation of the liquid core as the expected amplitudes of these terms are also hardly above the noise level of the astronomical results. The reasons for these limitations are mainly the atmospheric refraction, the variations in the direction of the vertical due to local tidal deformations, errors in the star catalogues, and the inevitable instrumental and personal errors.

Recently the possiblility of using close Earth satellites for determining the polar motion has been dramatically illustrated by the results obtained by Anderle and Beuglass (1970) from analysis of orbital residuals of Doppler observations. Comparison of their results with the results obtained by the Bureau International de l'Heure (Feissel et al., 1972) have indicated that the two methods give essentially equivalent results. The Doppler solution, moreover, gives the pole position every two days and more recently for each day compared to the BIH mean positions every five days.

The Doppler data have an accuracy of about 5 to $10 \mathrm{~m}$ (Anderle, 1970) but the important characteristic of the tracking system is the large number of stations capable of observing the satellite each time it passes over a station, irrespective of cloud cover. The laser range tracking of satellites offers considerable improvements in the determination of the motion of the Earth about its mass center because of its potentially high accuracy. Accuracies of between 1 and $5 \mathrm{~m}$ are now routinely collected to the seven satellites carrying retro-reflector in orbit and accuracies of about $10 \mathrm{~cm}$ or even better are technically feasible. Laser systems with these high accuracies will probably be in routine operation within a few years and the 'Cannonball' satellite proposed by the Smithsonian Astrophysical Observatory (Weiffenbach and Hoffman, 1970) will be particularly important for future work.

The advantages of satellite methods over classical astronomical methods are that, in the case of laser observations, the atmosphere has little influence on the results as 
it can be quite simply and adequately corrected for. The Earth tides are also less important and can be corrected for as implied by the results of Kozai (1968) and Newton (1968). On the other hand, all unmodelled orbital perturbations of gravitational and non-gravitational origin will influence the results obtained for the Earth motion.

The advantages of the laser methods over the Doppler systems now used are the greater accuracy of the former and the fact that the satellites used are entirely passive. They need only be equipped with a suitable array of corner cube reflectors and can be used for extremely long time periods without introducing discontinuities into the pole position results. A major problem with the laser tracking is that the system is dependent on the weather although day-light observations are possible. This limitation becomes less important for high and dense satellites, such as Cannonball, which have a greater orbital stability than closer satellites.

The equations of motion used for describing the motion of the satellite are, in principle, with respect to an inertial reference frame defined by the mean equator and the mean equinox at some specified epoch. The coordinates of the tracking stations rotating with the Earth should also be reduced to such an inertial frame using the transformation matrices describing the Earth's rotation, polar motion, precession and nutations. If any of the physical parameters describing these transformations are inadequate, discrepancies will occur between the theory describing the satellite motion and the observations of the satellite positions. These discrepancies then furnish the necessary information for improving the values of the parameters sought. The parameters of interest here are the coordinates of the instantaneous axis of rotation with respect to some fixed in the Earth origin, (for example by amplitude $\psi$ and azimuth $A$ ).

The observed discrepancies can be related to these pole coordinates in a variety of ways and the simplest is perhaps in terms of the orbital elements $i$ (inclination) $u$ (argument of latitude) and $\Omega$ (longitude of the ascending node). If the polar motion is precisely known these elements will refer to the desired inertial frame, all other perturbations being neglected for the moment. However, if the pole coordinates are unknown, the computed orbital elements $i^{\prime}, u^{\prime}$, and $\Omega^{\prime}$ will refer to a moving equator that is inclined to the inertial one. The differences between the two sets of elements are related to the pole coordinates by the following expressions (see also Figure 1)

$$
\begin{aligned}
& \Delta i=i^{\prime}-i=-\psi \sin \left(\Omega^{\prime}-\theta-A\right) \\
& \Delta u=u^{\prime}-u=\psi \cos \left(\Omega^{\prime}-\theta-A\right) \operatorname{cosec} i^{\prime} \\
& \Delta \Omega=\Omega^{\prime}-\Omega=\psi \cos \left(\Omega^{\prime}-\theta-A\right) \operatorname{cotan} i^{\prime}
\end{aligned}
$$

where $\theta$ is the sidereal angle.

Thus the three elements will exhibit periodic perturbations with periods of $\left(\Omega^{\prime}-\theta-A\right)$, or approximately $24 \mathrm{hr}$. For orbits computed from data collected over an integral number of these periods the mean values of $i^{\prime}, u^{\prime}$ and $\Omega^{\prime}$ would be zero and instantaneous angular elements would show the periodic variations from the mean as described by the above equations. 


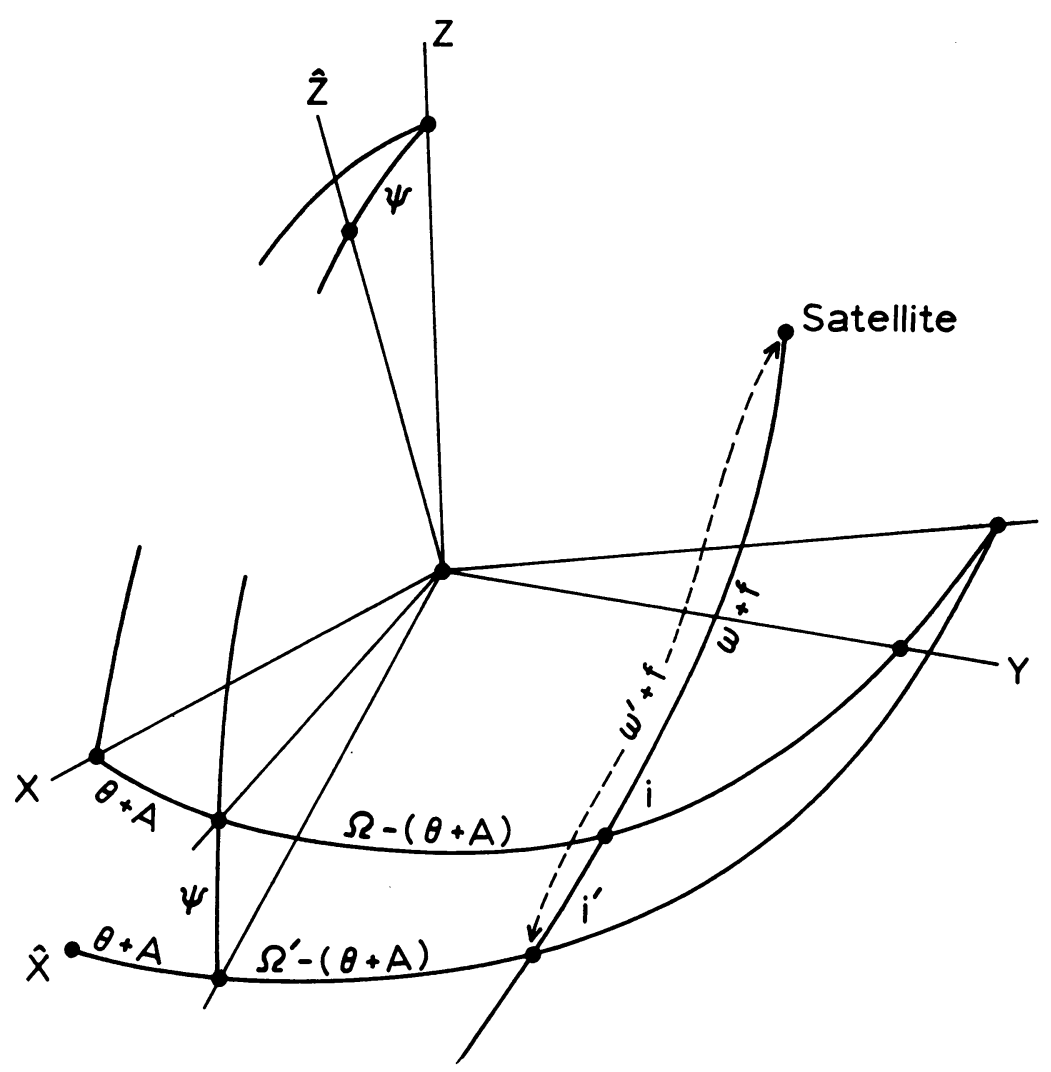

Fig. 1. Relationship between the orbital elements i, $\omega, \Omega$, referred to the inertial system $X Y Z$ and the elements $\mathrm{i}^{\prime}, \omega^{\prime}, \Omega^{\prime}$, referred to the quasi-inertial system $\not Y \mathcal{Z}$ resulting from the neglect of the polar motion.

In the above expressions small additional terms should be included because the computed elements $i^{\prime}, u^{\prime}$ and $\Omega^{\prime}$ do not refer to an inertial system. We should also consider the perturbations introduced into the orbital motion owing to the noncoincidence of the instantaneous principal axis of maximum inertia with the origin fixed in the Earth. Both perturbations, however, are quite small, less than $20 \mathrm{~cm}$, for the Geos and Transit type satellites. The latter perturbation is of particular interest as the motion of the maximum inertia axis can not be detected by classical astronomical methods.

The origin of the pole coordinates determined by the satellite method depends on the coordinates of the tracking stations used in the analysis. In the recent solution by Gaposchkin and Lambeck (1971) for example, the $z$-axis is tacitly assumed to coincide with the International Polar Motion Service origin since the pole coordinates provided by this service have been used in the analysis. But it is conceivable that small systematic errors in the station coordinates exist and that these may cause small departures of the $z$-axis from the assumed origin. This becomes particularly true when 
the axis is defined by a subset of station coordinates only; that is, when in the orbital analysis for the polar motion, only observations from some of the stations defining the coordinate system are used.

The basic assumption made so far is that the satellite orbits can be calculated without any errors. Unfortunately this is not so. In any orbit computations there will always be errors owing to inadequacies in the theories for modelling the various perturbing forces and there are always errors in the observational data. We can only hope to reduce these uncertainties to a level of insignificance.

The first type of error is of a secular nature. These may be caused by inadequacies in the even zonal harmonics of the geopotential or they may be caused by inadequacies in the models for air drag and solar radiation pressure. Errors of the second type are those with long periods. These may be due to the zonal terms, to the use of a quasiinertial reference frame for the orbital calculations, to resonances caused by certain longitude dependent terms in the geopotential, or to non-gravitational forces. The third type of error has short periods; these are equal to or less than those caused by the polar motion. The principal causes of these are uncertainties in the tesseral harmonics of the geopotential.

These various sources of error can be reduced by using more rigorous mathematical models for the various perturbing forces and by an improved knowledge of the physical parameters. They can also be minimized by a choice of satellite orbits that are less susceptible to these perturbations, and by a choice of physical satellite parameters that make the non-gravitational perturbations both smaller and more predictable. The proposed 'Cannonball' satellite, for example, satisfies both the orbital and the physical parameter requirements for compatibility with ultimate orbital accuracies of a few centimeters.

In general, if the errors in the orbital calculations have a frequency of $\alpha$ revs/day, the errors introduced into the pole coordinates are periodic with a frequency of $\alpha \pm \Omega$. Usually the frequencies of any likely source of error are known so that it is possible to filter out these effects from the pole coordinates provided that they are sufficiently distinct from the frequencies in the polar motion spectrum. A more detailed discussion of the various sources of error and their influence on the pole coordinates is given by Lambeck (1971).

An attempt has been made to deduce the pole positions from laser range data collected during April and May 1969 for the two Geos satellites. The results unfortunately were quite negative because of the poor distribution of the observations along the orbit and because of some systematic timing errors found in the data from one station. The need for a good distribution of the data along the orbit is the most important requirement particularly if a high resolution is sought; for example, pole positions every 12 hours. This means that the lasers must be capable of tracking the satellites during night and day, weather permitting. An other important requirement is that the distribution of observations by station be as uniform as possible in order to minimize the influence of any uncertainties in the station coordinates. With the data now being collected during the International Satellite Geodesy Experiment, these 
conditions are being partially satisfied and it appears that it will be possible to compute the pole positions with an accuracy of better than a meter and with a resolution of about two days. Better results, accuracies of about $20 \mathrm{~cm}$ and resolution of about $12 \mathrm{hr}$, will probably not be achieved until a special satellite such as the proposed Cannonball is launched.

\section{References}

Anderle, R. J.: 1970, 'Polar Motion Determinations by U.S. Navy Doppler Satellite Observations', N.W.L. Technical report TR-2432.

Anderle, R. J. and Beuglass, L. K.: 1970, Bull. Géodes. No. 96.

Feissel, M., Guinot, B., and Taton, N.: 1972, this volume, p. 104.

Gaposchkin, E. M. and Lambeck, K.: 1971, J. Geophys. Res. 76, 4855.

Guinot, B.: 1970, in L. Mansinha et al. (eds.), Earthquake Displacement Fields and the Rotation of the Earth, D. Reidel Publ. Comp., p. 54.

Kozai, Y.: 1968, Publ. Astron. Soc. Japan 20, 24.

Lambeck, K.: 1971, Bull. Géodes. No. 101, 263.

Newton, R. R.: 1968, Geophys. J. 14, 505.

Weiffenbach, G. C. and Hoffman, I. E.: 1970, Smithsonian Astrophys. Obs. Special Report, No. 329. 